\title{
Pluripotent stem cells reveal the developmental biology of human megakaryocytes and provide a source of platelets for clinical application
}

\author{
Naoya Takayama $\cdot$ Koji Eto
}

Received: 31 January 2012/Revised: 22 March 2012 / Accepted: 5 April 2012 / Published online: 24 April 2012

(C) The Author(s) 2012. This article is published with open access at Springerlink.com

\begin{abstract}
Human pluripotent stem cells [PSCs; including human embryonic stem cells (ESCs) and induced pluripotent stem cells (iPSCs)] can infinitely proliferate in vitro and are easily accessible for gene manipulation. Megakaryocytes (MKs) and platelets can be created from human ESCs and iPSCs in vitro and represent a potential source of blood cells for transfusion and a promising tool for studying the human thrombopoiesis. Moreover, disease-specific iPSCs are a powerful tool for elucidating the pathogenesis of hematological diseases and for drug screening. In that context, we and other groups have developed in vitro MK and platelet differentiation systems from human pluripotent stem cells (PSCs). Combining this co-culture system with a druginducible gene expression system enabled us to clarify the novel role played by $c-M Y C$ during human thrombopoiesis. In the next decade, technical advances (e.g., high-throughput genomic sequencing) will likely enable the identification of numerous gene mutations associated with abnormal thrombopoiesis. Combined with such technology, an in vitro system for differentiating human PSCs into MKs and platelets could provide a novel platform for studying human gene function associated with thrombopoiesis.
\end{abstract}

Keywords Human ESCs · Human iPSCs · Disease-specific iPSCs $\cdot$ ES/iPS-sac $\cdot$ MK $\cdot$ Platelet . Transfusion

\footnotetext{
N. Takayama $\cdot$ K. Eto $(\bowtie)$

Clinical Application Department,

Center for iPS Cell Research and Application,

Kyoto University, 53 Kawahara-cho,

Shogoin, Sakyo-ku, Kyoto 606-8507, Japan

e-mail: kojieto@cira.kyoto-u.ac.jp

N. Takayama

e-mail: naoya.takayama@cira.kyoto-u.ac.jp
}

\section{Introduction}

Platelets are essential for hemostasis and thrombosis. They also play vital roles in wound repair, inflammation, neoangiogenesis, and tumor metastasis. In humans, approximately $1 \times 10^{11}$ platelets are produced each day, presumably through the cytoplasmic fragmentation of megakaryocytes (MKs), their precursor cells, and production can be significantly increased on demand [1]. MK and platelet numbers are thought to be primarily controlled by thrombopoietin (TPO), whose serum levels may be regulated in part through a process entailing its binding to TPO/c-MPL (the thrombopoietin receptor) on platelets and MKs and its subsequent internalization and degradation (sponge theory) $[2,3]$.

Platelet production requires the commitment of hematopoietic stem cells (HSCs) to the MK lineage, followed by proliferation of the progenitors and terminal differentiation. During maturation, the MK precursor loses its capacity to divide but increases its ploidy through a process called endomitosis or endoreduplication, and also develops a unique membrane complex called the demarcation membrane system, as well as various types of granules, including lysosomes, dense granules, and $\alpha$-granules. Fully mature MKs form an extensive internal demarcation membrane, which is continuous with the plasma membrane and serves primarily as a membrane reservoir for the formation of the precursors of cytoplasmic extensions called proplatelets $[4,5]$. These sequential processes are controlled through the orchestrated activities of numerous signaling molecules, microRNAs, transcriptional factors, and their downstream target genes. Various gene-targeted mouse models have contributed to the characterization of the linkage between the machinery of MK maturation and platelet release, and the roles played by the transcriptional factors involved [6-11]. 
It has become evident, however, that some human diseases are not fully recapitulated in genetically engineered mouse models. In that regard, several groups, including ours, have developed in vitro MK and platelet differentiation systems from human pluripotent stem cells (PSCs) [12-17]. Human PSCs, which are easily accessible for controlled and specific genetic manipulation, are a promising tool for studying transcriptional regulation within the defined constraints of human MK development or cell signaling within platelets, which are anucleate and thus not amenable to direct gene manipulation. Moreover, patient-specific-induced pluripotent stem cells (iPSCs) enable one to investigate the pathogenesis of disease or test drugs using in vitro estimation assays based upon specific cell-traced differentiation, without the need for human subjects. This review focuses on the study of human thrombopoiesis using in vitro differentiation systems for human PSCs.

\section{The study of human thrombopoiesis in vitro}

\section{Sources of MKs and platelets}

The rarity of MKs within bone marrow limits the use of bone marrow specimens for studies of megakaryopoiesis and platelet generation. For this reason, human thrombopoiesis has largely been studied using in vitro differentiation systems developed from human primary hematopoietic stem/progenitor cells derived from cord blood, bone marrow, and mobilized peripheral bloodderived CD34+ cells [18-21]. These systems have enabled each developmental step in the process of megakaryopoiesis to be resolved and examined, and have also contributed to our understanding of human platelet biogenesis. However, primary human hematopoietic cells are heterogeneous, reflecting the differences in their genetic backgrounds, which can lead to a lack of consistency in the results. Moreover, while platelets are not amenable to genetic manipulation, it is possible to manipulate MKs to investigate the molecules expressed in platelets, but the efficiency of genetic transduction in MKs and their progenitors is not high.

On the other hand, we previously showed the utility of an in vitro MK differentiation system derived from genetically manipulated mouse ESCs [22], which can be easily expanded and provides relatively homogeneous cells. This strategy also seemed applicable to the study of human thrombopoiesis using human PSCs, prompting us to establish an in vitro differentiation system with human PSCs that enables genetic manipulation for investigating human thrombopoiesis.
In vitro generation of $\mathrm{MKs}$ and platelets from human PSCs

The culture methods needed to maintain the pluripotency of mouse ESCs are now well established and were improved by the finding that administration of two inhibitor $(2 \mathrm{i})$ reagents (MAP kinase inhibitor and GSK3 $\beta$ inhibitor) stabilizes the ground state/naive state [23]. In addition, differentiation protocols are being developed for application to a variety of cells and tissues, and several groups have reported producing platelets and/or MKs in vitro [22, 24, 25]. The first report of MK generation from human ESCs was presented by Gaur et al. [26]. They differentiated human ESCs on OP9 cells, a bone marrow stromal cell line derived from mice lacking macrophage colony stimulating factor [27]. OP9 cells are useful feeder cells and support differentiation of both mouse and human ESCs into mature hematopoietic cells in vitro. Gaur's group observed that after 15-17 days of co-culture in the presence of TPO, ESC-derived MKs began to express specific surface antigens, including two lineage markers, cluster of differentiation (CD) 41a (CD41a; integrin $\alpha \mathrm{IIb}$ ) and CD42b $(\mathrm{GPIb} \alpha)$, but no platelets were detected in this system [26]. We also failed to generate large numbers of platelet-like particles from human ESCs by this basic protocol.

By testing various combinations of feeder cells, cytokines, and culture periods, we were able to develop a novel in vitro differentiation system in which platelet-generating MKs were generated from human ESCs [12]. When we cocultured human ESCs with 10T1/2 cells, a highly stable fetal mesenchymal cell line derived from a $\mathrm{C} 3 \mathrm{H}$ mouse strain [28], in the presence of vascular endothelial growth factor (VEGF), unique sac-like structures derived from the ESCs (ES-sacs) appeared on day 14 of culture.

While it is well known that conventional ESC differentiation protocol with feeder cells by Gaur et al. [26] require the reseeding procedures both on day 7 and 11 (Fig. 1a), there was no necessity of reseeding procedure for up to 14 days on the appearance of ES-sac structure in our protocol (Fig. 1b).

These ES-sacs, particularly their external layer, expressed several endothelial markers, including CD31, CD34, and VEGF-receptor 2. ES-sac also contained CD34+/CD43+/ $\mathrm{CD} 45+$ hematopoietic progenitors inside that exhibited hematopoietic colony-forming potential in semisolid culture and differentiated into a variety of mature cells, i.e., not only MKs capable of releasing platelets, but also erythrocytes, lymphocytes, macrophages, and granulocytes, under conditions towards specific lineage commitment.

On the other hand, hESC-derived colonies that did not form ES-sacs failed to differentiate into hematopoietic cells, indicating that the ES-sac concentrated multipotent hematopoietic progenitors inside. 
(A)

Conventional protocol (Gaur et al.)

$\begin{array}{llll}\text { d0 } & \text { d7 } & \text { d11 } & \text { d15-17 }\end{array}$

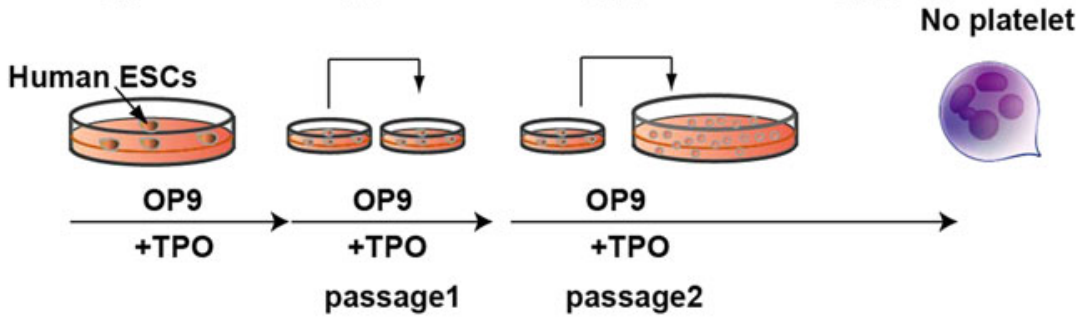

(B)

ES/iPS-sac method (Our protocol) d23-26

Single cells

Human ESCs

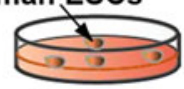

OP9 or $10 \mathrm{~T} 1 / 2$

+VEGF

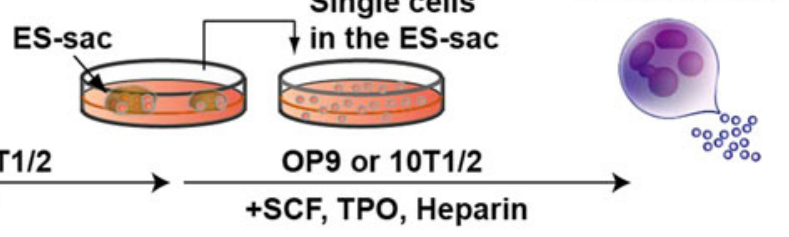

(C)

Hemangioblast/Blast colony method (Lu et al.)

d0 d4 d6-8 d10-14 d14-22

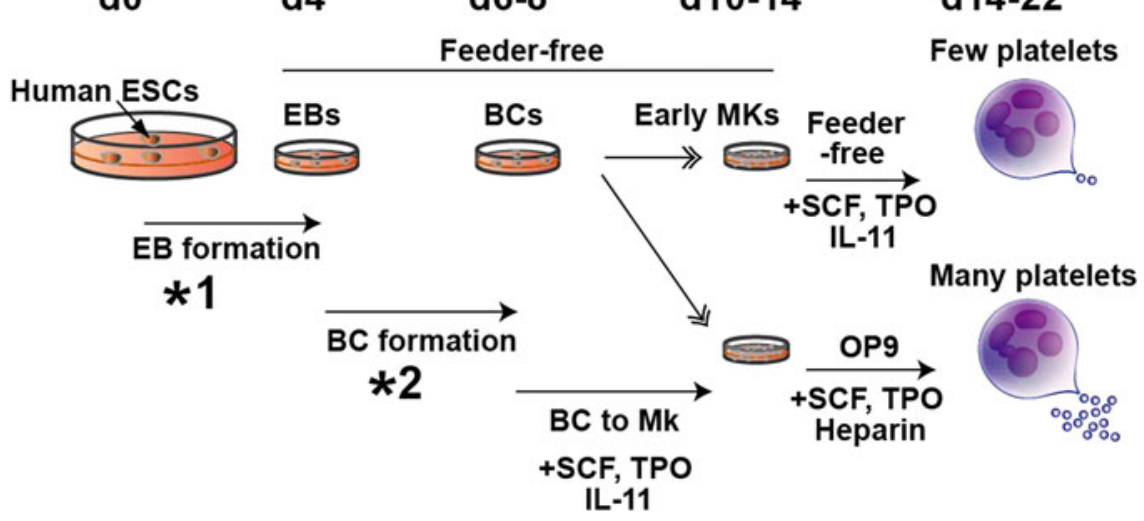

Fig. 1 Schematic diagrams of culture systems generating megakaryocyte and platelet from human pluripotent stem cells. a Conventional method (multi-round replating); hESCs are differentiated on OP9 stromal cells. On days 7 and 11, single cells derived from differentiated human ESC colonies are transferred onto fresh OP9 cells and further cultivated up to 17 days. In this culture system, platelets were hardly produced as reported. b ES/iPS-sac method; hESCs are co-cultured with 10T1/2 or OP9 stromal cells. On day 14 of culture, ESC/iPSC-derived sacs (ES/iPS-sac) are collected by using cell scraper and filtered with cell strainer to concentrate hematopoietic progenitors. The hematopoietic progenitors are then transferred onto fresh $10 \mathrm{~T} 1 / 2$ or OP9 stromal cells and cultivated up to day 26 . Megakaryocytes can be matured for producing platelets efficiently in

Early hematopoietic cells reportedly originate from hemangioblasts [29], which are a common precursor of both vascular endothelial cells and hematopoietic cells. ES-sacs this protocol. c Hemangioblast/Blast colony (BC) method; hESCs are transferred to ultra-low attached culture dishes in serum-free condition with cytokine combination (*1) for $\sim 4$ days leading to embryoid body (EB) formation. Single cells derived from EBs are again transferred to ultra-low attached culture dishes in serum-free condition with cytokine combination $(* 2)$ and $1 \%$ methylcellulose for 2-4 days towards promotion of blast colonies (BCs). BCs are differentiated to MK lineage cells in the presence of SCF, TPO, IL11 for 4-6 days. These steps are all feeder-free conditions. Plateletgeneration stage requires OP9 stromal cells as evidenced by efficient yield of platelets but not without OP9 stromal cells. (*1) BMP4, VEGF, SCF, TPO, and FLT3L, (*2) BMP4, VEGF, SCF, TPO, EPO, FLT3L, G-CSF, GM-CSF, and IL-6

may mimic the early hematopoietic organ, within which hematopoiesis and vasculogenesis occur simultaneously, and may also contain hematopoietic niche circumstances 
for hematopoietic stem cells (HSCs) and/or hematopoietic progenitor cells (HPCs).

Co-culture for an additional 7-11 days in the presence of $100 \mathrm{ng} / \mathrm{ml} \mathrm{TPO}, 50 \mathrm{ng} / \mathrm{ml}$ stem cell factor (SCF) and $25 \mathrm{U} / \mathrm{ml}$ heparin promotes differentiation of the hematopoietic progenitors within the ES-sacs into MKs [12, 13]. Of the cells, 50-60\% consistently express CD41a, CD42a, and $\mathrm{CD} 42 \mathrm{~b}$, which are essential and specific cell-surface molecules expressed on MKs and platelets [30, 31]. During MK maturation, a broad range of internal membrane systems, granules, and organelles are assembled [32-34], and the mature MKs contain an extensive internal demarcation membrane that serves as a membrane reservoir for later formation of proplatelets. In addition, an open canalicular system (OCS) for granule release is formed prior to the initiation of proplatelet assembly, so that when platelets are stimulated, the granules are released via the OCS [35]. We found a large number of proplatelet-forming MKs from day 22 to day 26 of culture, suggesting a successful development of demarcation membrane system (DMS) for platelet release and yield.

We also confirmed that various types of human iPSCs can be differentiated into mature MKs that release platelets in vitro [13], and we are presently confident that the ES/ iPS-sac method is a consistent and stable system for creating hematopoietic cells in vitro.

To establish xeno-free platelet generation system, Lu et al. have recently developed a unique feeder- and animal serum-free culture system in which 'hemangioblast' can be efficiently induced into hematopoietic progenitors [36], which in turn create erythrocytes [37], MKs, and platelets [15]. On the other hand, in such a system, adequate platelet production (yield) still must require known feeder cells OP9 mouse stromal cells, indicating that platelet release should be dependent upon feeder cells per se and/or critical factor(s) from feeder cells (Fig. 1c).

Development and maturation of MKs derived from human PSCs

The use of human HSCs has enabled the process of MK development to be characterized in detail [33]. The most primitive $\mathrm{MK}$ progenitors are the highly proliferative potential-colony-forming unit-MKs (HPP-CFU-MKs) and the burst-forming unit-MKs (BFU-MKs). BFU-MKs are capable of producing a more differentiated MK progenitor, the colony-forming unit-MK (CFU-MK), which can then differentiate into mature $\mathrm{MKs}$ that increase in size and ploidy through endomitosis. The maturation of MKs from HSCs is associated with changes in the expression of CD antigens, whereas MK progenitors (HPP-CFU-MK, BFU-MK, and CFU-MK) and immature MKs express CD34 [38], more mature MKs are negative for CD34 expression. The most widely used markers for examining MK differentiation are CD41a/CD61 and CD42 (GPIb/IX/V complex; the vWF receptor). CFU-MKs are the first $\mathrm{MK}$ lineage to have been identified based on a distinct surface phenotype that expresses CD41a/CD61 antigen. CD42 is expressed slightly later than CD41a/CD61 [38]; however, the expression of both correlate with MK maturity. Thus, during megakaryopoiesis from human HSCs, the CD34+CD41a+CD42+ and CD34-CD41a+CD42+ phenotypes represent more mature MKs, while CD34+CD41a+CD42- cells are intermediate, not fully mature, MKs.

To clarify the relationship between the development of MKs derived from human PSCs and the changes in CD antigens, we isolated and divided hematopoietic progenitors from ES-sacs into CD34+/CD41a-, CD34+/ CD41a+, CD34-/CD41a+, and CD34-/CD41a- subpopulations, and then allowed them to differentiate into MKs on 10T1/2 feeder cells or used RT-PCR to assess their gene expression. Most CD34+/CD41a+ and CD34-/ CD41a+ cells should be differentiated into CD41a+/ $\mathrm{CD} 42 \mathrm{~b}(\mathrm{GPIb} \alpha)+$ mature MKs, indicating both populations were committed to MK lineage.

On the other hand, $40 \%$ of hematopoietic cells derived from the CD34+/CD41a- population were MKs. This population generated multi-lineage myeloid colonies, such as erythrocyte, MKs, macrophage, or granulocytes, in colonyforming assay, indicating that the CD34+/CD41a- population contains the potential for differentiation into both megakaryocytic and other lineages. Using RT-PCR, we confirmed that CD34+/CD41a- progenitors can develop into CD41a+ populations. In addition, the CD34+/CD41a+ cells, but not CD34+/CD41a- cells, also expressed GATA-1, FOG-1, Fli-1, and NF-E2, which are required for megakaryopoiesis [39]. These findings are consistent with the developmental behavior observed during megakaryopoiesis from HSCs within bone marrow or cord blood [40, 41].

Characterization of human ESC/iPSC-derived platelets in vitro

When we examined the surface markers of human ESC/iPSCderived platelet-like particles using flow cytometry with the same forward- and side-scatter gates suitable for fresh adult human platelets, we found that the culture supernatants contained CD41a+ particles, which also expressed other functional receptors normally found on platelets, including CD42a (GPIX), CD42b (GPIb $\alpha$ ), and GPVI. About half of the $\mathrm{CD} 41 \mathrm{a}+$ platelets generated in vitro lost CD42b $(\mathrm{GPIb} \alpha)$ due to cleavage of its extracellular N-terminal region by a disintegrin and metalloproteinase (ADAM) 17 (also referred to as tumor necrosis factor $\alpha$ converting enzyme, TACE) [42], though GPIb $\alpha$ could be restored by addition of a metalloproteinase inhibitor to the culture [13]. 
Electron microscopic examination of the cytosolic structures of human ESC/iPSC-derived platelets revealed their morphology to be similar to that of human peripheral blood-derived platelets, with normal microtubules, granules, and OCS. These platelets also displayed integrin $\alpha \operatorname{IIb} \beta 3$ (CD41a/CD61 complex) activation, which is essential for platelet aggregation, and they spread in response to the physiological agonists ADP or thrombin. Human ESC/iPSC-derived platelets thus appear structurally and functionally intact in vitro, but the most important function of platelets in vivo is hemostasis and thrombus formation under flow conditions. Earlier in vivo imaging was capable of detecting only mass platelet aggregation [43]. However, to visualize the behavior of individual platelets upon initiation of adhesion to an injured vessel wall and the subsequent steps in thrombus formation under flow conditions within vessels, we recently developed a novel in vivo imaging system that employs high-spatiotemporal resolution confocal laser microscopy [44, 45]. With our system, intravenous administration of hematoporphyrin followed by laser exposure induces rapid formation of a microthrombus. In these experiments, iPSCplatelets stained with tetramethylrhodamine ethyl ester, a membrane-permeant, cationic, red-orange fluorescent dye that is readily sequestered by active mitochondria, were transfused into a NOG (NOD/Shi-scid, IL-2R $\gamma$ null) mouse with thrombocytopenia, where red staining then indicated living iPSC-platelets. Following laser-induced vessel injury, we clearly observed that iPSC-platelets initially adhered to the injured vessel wall, coordinating with host platelets, and that this process ultimately led to thrombus formation and vessel occlusion, which suggests the iPSCderived platelets are functionally intact in vivo [13]. Similarly, using a conventional imaging system that enabled them to detect mass aggregations, Lu et al. [15] observed that human ESC-derived platelets contributed to thrombus formation in living mice. Collectively then, these findings indicate that platelets generated from human PSCs in vitro share many, if not all, of the characteristics of human peripheral blood-derived platelets. Using human iPSC specimens, we also confirmed that human iPSCs created from dermal fibroblasts through artificial reprogramming could be differentiated in vitro into functional platelets that could serve to supply HLA-identical platelets, or perhaps the use of disease-specific iPSCs could provide new information able to serve as a platform for the study of abnormal thrombopoiesis.

In vitro analysis of the genes for platelet biogenesis using PSCs with gene manipulation

Megakaryopoiesis is a complex process governed by the orchestrated activities of numerous molecular mediators, especially transcriptional factors, signaling molecules, microRNAs, and the products of their various target genes. Several gene-targeted mouse models have provided important information about thrombopoiesis, but the generation of gene-targeted mice is both time-consuming and expensive; moreover, it is sometimes embryonically lethal before the onset of hematopoiesis, which makes it difficult to analyze the MK lineage. In that context, several groups, including ours, previously showed the advantages of in vitro differentiation of MKs from mouse ESCs over genetargeted mouse models for analysis of functional molecules in MKs and platelets [22, 46-48].

The Wiskott-Aldrich syndrome protein (WASp) homolog Wave (WASp family Verprolin-homologous protein) functions downstream of Rac, a member of the Rho family of small GTPases, and plays a pivotal role in lamellipodia formation in cells. Wave2-null mice die by embryonic day 11.5 or 12.5 due to a defect in vascular development. We therefore generated Wave2-/- mouse ESCs from which we then derived MKs using our in vitro differentiation culture system. The Wave2-/- MKs generated in this way showed severely impaired terminal differentiation, platelet production, and agonist-induced peripheral lamellipodia formation on fibrinogen. We also confirmed that using small interfering RNA (siRNA) to knock down Abl-interactor (Abi)1, which forms a complex with Wave2, produces a phenotype that is striking similar to Wave2-/$\mathrm{MKs}$; that is, both their maturation and integrin $\alpha \operatorname{IIb} \beta 3$ mediated spreading were impaired. This is indicative of the indispensable role played by the Wave2/Abi1 complex in MK maturation and lamellipodia formation [46, 49].

It is known that the genetic mutations in some mouse models fully recapitulate the phenotype of the corresponding human disease. This is not always the case, however. In fact, mutation or deletion of several genes (e.g., NFE2, Wave2, and Spectrin) known to be important for thrombopoiesis in mouse models, have not been identified in patients with thrombocytopenia $[8,46,50]$. Consequently, we are unable to validate the function of these genes in human cells. Fortunately, recent developments with human ESCs [51] and iPSCs [52-54] and with gene-targeting technology enable us to provide an alternative tool for the study of human hematopoietic ontology and lineage commitment. For example, we used gene manipulation with human iPSCs to characterize the role played by $c$ $M Y C$ in human megakaryopoiesis. It had already been reported that $c-M y c$ plays an essential role in both embryonic and adult hematopoiesis, though its effects on megakaryopoiesis and thrombopoiesis in various mouse models remained unclear [55-58]. Two studies of the effects of inducible $c-M y c$ overexpression on the control of MK-specific differentiation showed that $c-M y c$ exerts a positive effect on the proliferation of MK progenitors [55, 56]. Moreover, $c-M y c$ is reportedly essential for the TPO-c-mpl axis in megakaryopoiesis [57]. On 
the other hand, more recent studies using $c$-Myc-deficient mice showed that the absence of the gene actually leads to a cellautonomous increase in the number of low-ploidy MKs and platelets [58]. The reasons for this apparent discrepancy are still not completely clear. However, by comparing the efficiency of MK and platelet generation from several iPS clones established using retroviral vectors harboring either four (OCT3/4, SOX2, KLF4 and c-MYC) or three (without $c-M Y C$ ) reprogramming factors, we demonstrated that although $c$ $M Y C$ is beneficial for MK proliferation, its sustained expression inhibits platelet release. This idea was confirmed by additional experiments showing that overexpression of $c$ $M Y C$, but none of the other reprogramming factors (OCT3/4, $S O X 2, K L F 4$ ), recapitulated the time course of the enhanced megakaryopoiesis. In addition, flow cytometry revealed that most of the cells in specimens overexpressing $c-M Y C$ were CD41a+GPIb $\alpha+$, but cells in the other specimens were not, though only mononuclear and lower-ploidy cells were present. The numbers of platelets per MK, one indicator of MK maturation, were quite low in specimens overexpressing $c-M Y C$, and the released platelet-like particles did not express GPIb $\alpha$, suggesting that MKs from specimens overexpressing $c-M Y C$ were immature. Interestingly, the phenotype of platelets generated from MKs overexpressing $c-M Y C$ was similar to that in Gata1-/- mouse models, which showed low expression of GPIb $\alpha$, abnormal ultrastructure, and defective integrin $\alpha \operatorname{IIb} \beta 3$ activation in response to thrombin or ADP [59].

The results summarized above led to us to conclude that excessive and sustained expression of $c-M Y C$ in human ESCs may promote lineage commitment into megakaryopoiesis without maturation. Moreover, overexpression of $c$ $M Y C$ in hematopoietic cells to levels that are inhibitory for platelet generation activates expression of the senescence/ apoptosis-inducing p14 (ARF) and pl6 (INK4A) genes, leading to senescence and apoptosis without maturation
(Fig. 2). Thus, there appears to be a narrow window of $c$ $M Y C$ expression for efficient platelet development.

To further confirm whether an increase and subsequent decline in $c-M Y C$ is critical for megakaryopoiesis leading to an efficient platelet yield, we prepared a Sendai viral vector $(\mathrm{SeV})$ harboring the four reprogramming genes; this enabled RNA viral transduction during the generation of human iPSCs without integration of DNA into the chromosome [60]. Thereafter, a doxycycline (DOX)-inducible $c-M Y C$ overexpression system in a lentiviral vector was applied to the SeV-based human iPSCs. This approach enabled us to confirm that transient up-regulation of $c-M Y C$ expression at the level of the MK progenitors and its subsequent decline increased the total numbers of mature MKs, proplatelets and GPIb $\alpha+$ platelets [13].

Studying thrombopoiesis using patient-specific iPSCs

Our culture system enables us to investigate in detail the developmental stages of MKs and platelets derived from human iPSCs. Moreover, MKs derived from disease-specific human iPSCs represent a powerful tool for investigating the unresolved aspects of the mechanisms underlying thrombocytopenia and for screening novel therapeutic agents for patients suffering from reduced platelet production and/or their impaired function (Fig. 3). This is illustrated in the following example. Congenital amegakaryocytic thrombocytopenia (CAMT) is an autosomal recessive disorder caused by the loss of function of $c-M P L$, the gene encoding the TPO receptor. It presents at birth with severe thrombocytopenia and evolves into fatal bone marrow failure in the early years of life [61]. A $c$ $\mathrm{Mpl}-/-$ mouse model failed to fully recapitulate the disease phenotype; $c-M p l-/-$ mice showed sustained thrombocytopenia and reduced numbers of marrow cells
Fig. 2 Proposed model of thrombopoiesis by $c-M Y C$. We observed at least two distinct patterns of megakaryopoiesis in association with $c-M Y C$ kinetics. One is that excessive and sustained expression of $c$ $M Y C$ in MKs induces INK4A and $A R F$, leading to senescence and apoptosis without maturation of MKs. Another is that a decline in the $c-M Y C$ expression after a transient increase is a hallmark for MKs that production of functional $\mathrm{GPIb} \alpha+$ platelets is apparently detectable

\section{Role of c-MYC on human thrombopoiesis}

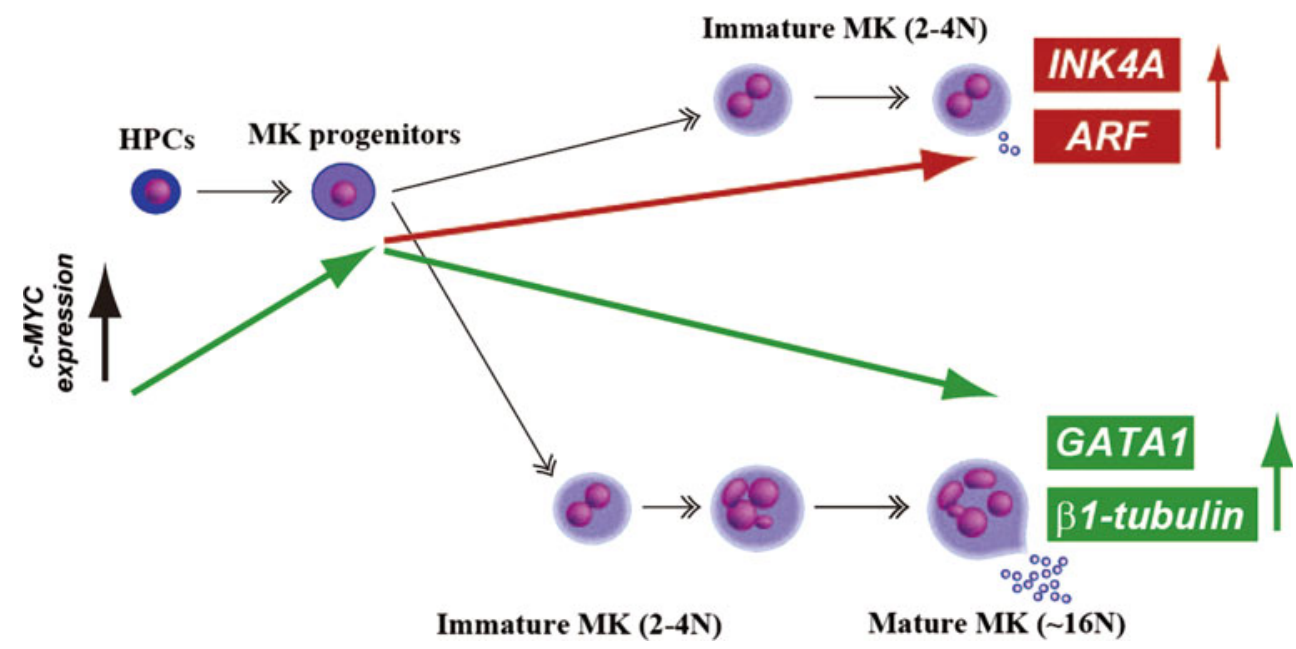


Fig. 3 Disease modeling of thrombopoiesis using patientspecific iPSCs. iPS cell technology enables us to model human disease in vitro. Diseasespecific iPSCs can be established from somatic cells in an individual patient by introduction of the defined reprogramming factors. Combing with in vitro differentiation system, genetic and epigenetic studies would reveal the pathogenesis of impaired megakaryocyte development in defined developmental steps. Novel drug screening for platelet disorders will also be applicable by using this system

\section{Disease modeling of thrombopoiesis using patient-specific iPSCs}

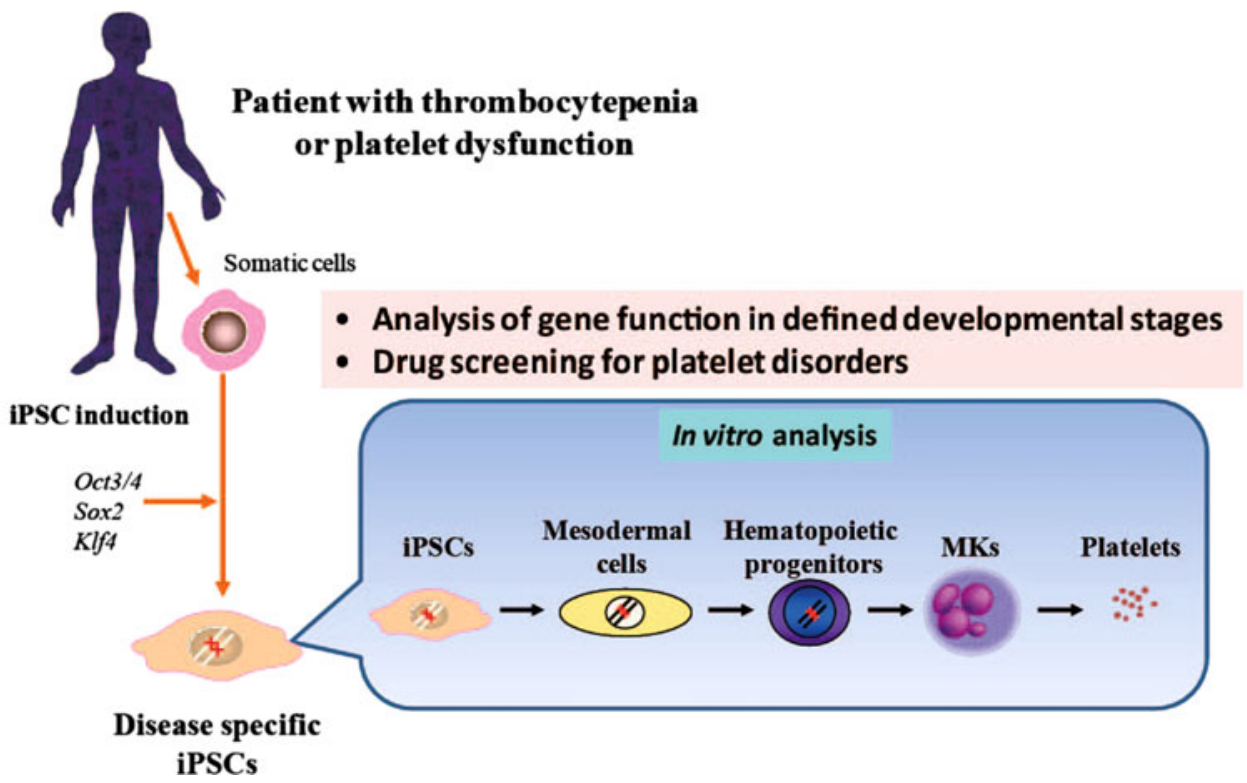

throughout their lives, but they survived to old age without developing bone marrow failure. To clarify the differential phenotype caused by the loss of MPL between humans and mice, we established iPSCs derived from skin fibroblasts collected from a CAMT patient treated with curative bone marrow transplantation. We then used our in vitro culture system to obtain hematopoietic progenitors to evaluate the function of $M P L$ during the early and late phases of human hematopoiesis [62]. This patient's iPSCs provided valuable insight into the mechanism of CAMT development.

Application of human PSC-derived platelets for future transfusion medicine

\section{Proof of concept: the stable supply of HLA-identical platelet concentrates}

It is well known that repeated transfusion induces production of antibodies against allogenic HLA on the transfused platelets [63], which renders the patient unresponsive to platelet transfusion therapy. To avoid such immune reactions following multiple allogeneic platelet transfusions, it is desirable to use HLA-matched or autologous platelets. In this context, we propose that the use of HLA-matched human iPSCs may be an effective strategy for generating functional platelets for patients requiring repeated transfusion. We have shown that iPSC-derived platelets are functional in vivo and could represent a novel solution for the shortage of donated platelets [13]. Now we attempt to modify the culture system to provide huge amount of platelets for clinical application.
Preservation of platelet function in vitro using metalloproteinase inhibitor

The functional platelet paradigm in hemostasis and thrombosis is the initiation of platelet adhesion to the extracellular matrix [64]. Key events in that process are the interaction between GPIb $\alpha$ and vWF present in the extracellular matrix [64], as well as the simultaneous interaction between surface-bound collagen and the platelet receptors GPVI and integrin $\alpha 2 \beta 1$ [65]. The net result is activation of integrin $\alpha \mathrm{IIb} \beta 3$, which turns into a competent fibrinogen receptor and leads to the formation of platelet aggregates [64]. Notably, aged platelets show a tendency to shed GPV and the extracellular domain of GPIb $\alpha$, which contains the binding sites for vWF and thrombin [66, 67]. This phenomenon makes it difficult to preserve platelet concentrates for more than 7 days.

The shedding process likely involves the action of ADAM 17 and leads to a reduction in platelet function [42, 68]. We observed that, in culture, mouse ESC-derived platelets shed the extracellular domains of GPIb $\alpha$ and GPV, as well as GPVI, possibly through the action of ADAM10, which reduces $\alpha \operatorname{IIb} \beta 3$ activation and actin polymerization and impairs thrombus formation. However, administration of the metalloproteinase inhibitor GM6001 during differentiation increased the expression of GPIb $\alpha$ and GPVI and improved both thrombogenesis in vitro and post-transfusion recovery in vivo [25]. Administration of GM6001 also inhibited shedding of the extracellular domain of GPIb $\alpha$ from human iPSC-derived platelets [13]. These data suggest that regulation of metalloproteinases in culture could 
be useful for obtaining high-quality and efficacious human iPSC-derived platelets for transfusion.

\section{Conclusions and future directions}

Several gene-targeted mouse models have provided important information about thrombopoiesis. While the genetic interventions, i.e., mutations by mouse models recapitulate the phenotype of the corresponding human disease, some of the models fail to show the mirror of human diseases. Of note from recent important work is that there is the difference of ortholog-matched mRNA expression between mouse and human platelets by using RNA sequence technology. This study showed that 4,990 mRNAs were commonly expressed both in human and mouse platelets, but 3,592 mRNAs were specific for human platelets and 1,022 mRNAs were specific for mouse platelets. They are associated with previously described functional disparities between mouse and human platelets at the transcript level, including protease activated receptor-1 (PAR1) [36, 69], protease activated receptor-3 (PAR3) [70], and platelet activating factor receptor (PAF-R) [71]. These strongly indicate the value of the study regarding human thrombopoiesis by human hematopoietic cells. In general, human primary hematopoietic stem/progenitor cells derived from cord blood, bone marrow, or peripheral blood are a main resource. We herein propose human PSCs as an alternative source.

MKs generated from human PSCs in vitro have the potential to form proplatelets that function like those derived from human HSCs, and the platelets released are functionally comparable to human peripheral bloodderived platelets. The in vitro generation of $\mathrm{MKs}$ and platelets from human PSCs has four advantages over primary HSCs. First, human PSCs are pluripotent cells that can proliferate almost indefinitely in vitro, thereby stably providing experimental samples that yield reproducible results. By contrast, inescapable variation in genetic background for each experiment makes it difficult to obtain consistent results with primary HSCs derived from cord blood or bone marrow. Second, human PSCs are a promising cell source for studying the ontogeny of blood cells in defined steps. It has been well demonstrated in mouse models that HSCs can provide all types of blood cells throughout life. Blood cells arise at the yolk-sac and transit to the AGM, fetal liver, and bone marrow during ontogeny [72-74]. In humans, however, the development of hematopoietic cells has not been characterized because of the difficulty in obtaining samples from human embryos. Human PSCs are an effective tool that enables one to recapitulate human ontology in vitro by appropriately defining the culture conditions, as we have shown. Third, human PSCs are easily amenable to gene manipulation.
Megakaryopoiesis is a complex process governed by the orchestrated activities of numerous molecular mediators, especially transcriptional factors, signaling molecules, microRNAs, and the products of their various target genes. Many transcriptional factors including Ets1, Runx1, Scl/ tal1, Gata1, NFE2, Mef2C, MafG, and MafK are involved in megakaryopoiesis [75-77].

Megakaryopoiesis is also controlled by a lot of microRNAs by regulating the expression of these target genes [71]. To study the unknown role of transcriptional factors or microRNAs on human thrombopoiesis, human PSCs should be a useful tool. For example, we were able to demonstrate the novel function of $c-M Y C$ on human thrombopoiesis by using in vitro differentiation of human PSCs with gene manipulation.

Fourth, patient-specific iPSCs facilitate the study of disease mechanisms and drug screening [78]. Although gene-targeted mouse models have provided important information on the pathogenesis of human disease, some human diseases are not completely recapitulated in these models. For example, to clarify the function of $c-M P L$, the TPO receptor, we established iPSCs from skin fibroblasts collected from a patient with CAMT. In vitro differentiation of CAMT-iPSCs revealed the critical role played by $c$ $M P L$ during early human thrombopoiesis. We anticipate that future technical advances in genome sequencing and/ or epigenome analysis will enable identification of numerous gene mutations associated with congenital thrombocytopenia or megakaryocytic leukemia. When that happens, disease-specific iPSCs would be a powerful tool for distinguishing pathogenic gene mutations from the many neutral mutations. Finally, establishment of an in vitro system for platelet generation from human iPSCs could usher in a new era of platelet transfusion therapy. Platelets generated from human iPSCs in vitro show normal function during thrombus formation in living mice [13], but a more efficient system for platelet generation will be required for future clinical applications.

Acknowledgments We thank Dr. S. Kunishima for providing valuable discussion and apologize to researchers whose work could not be cited because of space limitations. We declare no competing financial interests.

Open Access This article is distributed under the terms of the Creative Commons Attribution License which permits any use, distribution, and reproduction in any medium, provided the original author(s) and the source are credited.

\section{References}

1. Branehog I, Ridell B, Swolin B, Weinfeld A (1975) Megakaryocyte quantifications in relation to thrombokinetics in primary 
thrombocythaemia and allied diseases. Scand $\mathrm{J}$ Haematol 15:321-332

2. Kuter DJ, Rosenberg RD (1995) The reciprocal relationship of thrombopoietin (c-Mpl ligand) to changes in the platelet mass during busulfan-induced thrombocytopenia in the rabbit. Blood 85:2720-2730

3. Nagasawa T, Hasegawa Y, Shimizu S, Kawashima Y, Nishimura S et al (1998) Serum thrombopoietin level is mainly regulated by megakaryocyte mass rather than platelet mass in human subjects. Br J Haematol 101:242-244

4. Italiano JE Jr, Lecine P, Shivdasani RA, Hartwig JH (1999) Blood platelets are assembled principally at the ends of proplatelet processes produced by differentiated megakaryocytes. J Cell Biol 147:1299-1312

5. Junt T, Schulze H, Chen Z, Massberg S, Goerge T et al (2007) Dynamic visualization of thrombopoiesis within bone marrow. Science 317:1767-1770

6. Shivdasani RA, Fujiwara Y, McDevitt MA, Orkin SH (1997) A lineage-selective knockout establishes the critical role of transcription factor GATA-1 in megakaryocyte growth and platelet development. EMBO J 16:3965-3973

7. Tsang AP, Fujiwara Y, Hom DB, Orkin SH (1998) Failure of megakaryopoiesis and arrested erythropoiesis in mice lacking the GATA-1 transcriptional cofactor FOG. Genes Dev 12:1176-1188

8. Lecine P, Villeval JL, Vyas P, Swencki B, Xu Y et al (1998) Mice lacking transcription factor NF-E2 provide in vivo validation of the proplatelet model of thrombocytopoiesis and show a platelet production defect that is intrinsic to megakaryocytes. Blood 92:1608-1616

9. Onodera K, Shavit JA, Motohashi H, Yamamoto M, Engel JD (2000) Perinatal synthetic lethality and hematopoietic defects in compound mafG:mafK mutant mice. EMBO J 19:1335-1345

10. Spyropoulos DD, Pharr PN, Lavenburg KR, Jackers P, Papas TS et al (2000) Hemorrhage, impaired hematopoiesis, and lethality in mouse embryos carrying a targeted disruption of the Fli1 transcription factor. Mol Cell Biol 20:5643-5652

11. Pang L, Xue HH, Szalai G, Wang X, Wang Y et al (2006) Maturation stage-specific regulation of megakaryopoiesis by pointed-domain Ets proteins. Blood 108:2198-2206

12. Takayama N, Nishikii H, Usui J, Tsukui H, Sawaguchi A et al (2008) Generation of functional platelets from human embryonic stem cells in vitro via ES-sacs, VEGF-promoted structures that concentrate hematopoietic progenitors. Blood 111:5298-5306

13. Takayama N, Nishimura S, Nakamura S, Shimizu T, Ohnishi R et al (2010) Transient activation of c-MYC expression is critical for efficient platelet generation from human induced pluripotent stem cells. J Exp Med 207:2817-2830

14. Klimchenko O, Mori M, Distefano A, Langlois T, Larbret $F$ et al (2009) A common bipotent progenitor generates the erythroid and megakaryocyte lineages in embryonic stem cell-derived primitive hematopoiesis. Blood 114:1506-1517

15. Lu SJ, Li F, Yin H, Feng Q, Kimbrel EA et al (2011) Platelets generated from human embryonic stem cells are functional in vitro and in the microcirculation of living mice. Cell Res 21:530-545

16. Takayama N, Eto K (2012) In vitro generation of megakaryocytes and platelets from human embryonic stem cells and induced pluripotent stem cells. Methods Mol Biol 788:205-217

17. Gekas C, Graf T (2010) Induced pluripotent stem cell-derived human platelets: one step closer to the clinic. J Exp Med 207:2781-2784

18. Debili N, Coulombel L, Croisille L, Katz A, Guichard J et al (1996) Characterization of a bipotent erythro-megakaryocytic progenitor in human bone marrow. Blood 88:1284-1296

19. Bornstein R, Garcia-Vela J, Gilsanz F, Auray C, Cales C (2001) Cord blood megakaryocytes do not complete maturation, as indicated by impaired establishment of endomitosis and low expression of G1/S cyclins upon thrombopoietin-induced differentiation. Br J Haematol 114:458-465

20. Mattia G, Vulcano F, Milazzo L, Barca A, Macioce G et al (2002) Different ploidy levels of megakaryocytes generated from peripheral or cord blood CD34+ cells are correlated with different levels of platelet release. Blood 99:888-897

21. Matsunaga T, Tanaka I, Kobune M, Kawano Y, Tanaka M et al (2006) Ex vivo large-scale generation of human platelets from cord blood CD34+ cells. Stem Cells 24:2877-2887

22. Eto K, Murphy R, Kerrigan SW, Bertoni A, Stuhlmann H et al (2002) Megakaryocytes derived from embryonic stem cells implicate CalDAG-GEFI in integrin signaling. Proc Natl Acad Sci USA 99:12819-12824

23. Ying QL, Wray J, Nichols J, Batlle-Morera L, Doble B et al (2008) The ground state of embryonic stem cell self-renewal. Nature 453:519-523

24. Fujimoto TT, Kohata S, Suzuki H, Miyazaki H, Fujimura K (2003) Production of functional platelets by differentiated embryonic stem (ES) cells in vitro. Blood 102:4044-4051

25. Nishikii H, Eto K, Tamura N, Hattori K, Heissig B et al (2008) Metalloproteinase regulation improves in vitro generation of efficacious platelets from mouse embryonic stem cells. J Exp Med 205:1917-1927

26. Gaur M, Kamata T, Wang S, Moran B, Shattil SJ et al (2006) Megakaryocytes derived from human embryonic stem cells: a genetically tractable system to study megakaryocytopoiesis and integrin function. J Thromb Haemost 4:436-442

27. Nakano T, Kodama H, Honjo T (1994) Generation of lymphohematopoietic cells from embryonic stem cells in culture. Science 265:1098-1101

28. Hiroyama T, Miharada K, Aoki N, Fujioka T, Sudo K et al (2006) Long-lasting in vitro hematopoiesis derived from primate embryonic stem cells. Exp Hematol 34:760-769

29. Choi K, Kennedy M, Kazarov A, Papadimitriou JC, Keller G (1998) A common precursor for hematopoietic and endothelial cells. Development 125:725-732

30. den Dekker E, van Abel M, van der Vuurst H, van Eys GJ, Akkerman JW et al (2003) Cell-to-cell variability in the differentiation program of human megakaryocytes. Biochim Biophys Acta 1643:85-94

31. Tomer A (2004) Human marrow megakaryocyte differentiation: multiparameter correlative analysis identifies von Willebrand factor as a sensitive and distinctive marker for early $(2 \mathrm{~N}$ and $4 \mathrm{~N})$ megakaryocytes. Blood 104:2722-2727

32. Schulze H, Shivdasani RA (2004) Molecular mechanisms of megakaryocyte differentiation. Semin Thromb Hemost 30: 389-398

33. Szalai G, LaRue AC, Watson DK (2006) Molecular mechanisms of megakaryopoiesis. Cell Mol Life Sci 63:2460-2476

34. Battinelli EM, Hartwig JH, Italiano JE Jr (2007) Delivering new insight into the biology of megakaryopoiesis and thrombopoiesis. Curr Opin Hematol 14:419-426

35. Patel SR, Hartwig JH, Italiano JE Jr (2005) The biogenesis of platelets from megakaryocyte proplatelets. J Clin Invest 115:3348-3354

36. Connolly AJ, Ishihara H, Kahn ML, Farese RV Jr, Coughlin SR (1996) Role of the thrombin receptor in development and evidence for a second receptor. Nature 381:516-519

37. Italiano JE Jr, Stewart M, Roberts TM (1999) Localized depolymerization of the major sperm protein cytoskeleton correlates with the forward movement of the cell body in the amoeboid movement of nematode sperm. J Cell Biol 146:1087-1096

38. Debili N, Issaad C, Masse JM, Guichard J, Katz A et al (1992) Expression of CD34 and platelet glycoproteins during human megakaryocytic differentiation. Blood 80:3022-3035 
39. Shivdasani RA (2001) Molecular and transcriptional regulation of megakaryocyte differentiation. Stem Cells 19:397-407

40. Debili N, Wendling F, Katz A, Guichard J, Breton-Gorius J et al (1995) The Mpl-ligand or thrombopoietin or megakaryocyte growth and differentiative factor has both direct proliferative and differentiative activities on human megakaryocyte progenitors. Blood 86:2516-2525

41. Schipper LF, Brand A, Reniers N, Melief CJ, Willemze R et al (2003) Differential maturation of megakaryocyte progenitor cells from cord blood and mobilized peripheral blood. Exp Hematol $31: 324-330$

42. Bergmeier W, Piffath CL, Cheng G, Dole VS, Zhang Y et al (2004) Tumor necrosis factor-alpha-converting enzyme (ADAM17) mediates GPIbalpha shedding from platelets in vitro and in vivo. Circ Res 95:677-683

43. Dubois C, Panicot-Dubois L, Gainor JF, Furie BC, Furie B (2007) Thrombin-initiated platelet activation in vivo is $\mathrm{vWF}$ independent during thrombus formation in a laser injury model. J Clin Invest 117:953-960

44. Takizawa H, Nishimura S, Takayama N, Oda A, Nishikii $\mathrm{H}$ et al (2010) Lnk regulates integrin alphaIIbbeta3 outside-in signaling in mouse platelets, leading to stabilization of thrombus development in vivo. J Clin Invest 120:179-190

45. Nishimura S, Manabe I, Nagasaki M, Kakuta S, Iwakura Y, et al. (2011) In vivo imaging visualizes discoid platelet aggregations without endothelium disruption and implicates contribution of inflammatory cytokine and integrin signaling. Blood

46. Eto K, Nishikii H, Ogaeri T, Suetsugu S, Kamiya A et al (2007) The WAVE2/Abi1 complex differentially regulates megakaryocyte development and spreading: implications for platelet biogenesis and spreading machinery. Blood 110:3637-3647

47. Frontelo P, Manwani D, Galdass M, Karsunky H, Lohmann F et al (2007) Novel role for EKLF in megakaryocyte lineage commitment. Blood 110:3871-3880

48. Chen Z, Naveiras O, Balduini A, Mammoto A, Conti MA et al (2007) The May-Hegglin anomaly gene MYH9 is a negative regulator of platelet biogenesis modulated by the Rho-ROCK pathway. Blood 110:171-179

49. Larson MK, Watson SP (2006) Regulation of proplatelet formation and platelet release by integrin alpha IIb beta3. Blood 108:1509-1514

50. Patel-Hett S, Wang H, Begonja AJ, Thon JN, Alden EC et al (2011) The spectrin-based membrane skeleton stabilizes mouse megakaryocyte membrane systems and is essential for proplatelet and platelet formation. Blood 118:1641-1652

51. Thomson JA, Itskovitz-Eldor J, Shapiro SS, Waknitz MA, Swiergiel JJ et al (1998) Embryonic stem cell lines derived from human blastocysts. Science 282:1145-1147

52. Takahashi K, Tanabe K, Ohnuki M, Narita M, Ichisaka T et al (2007) Induction of pluripotent stem cells from adult human fibroblasts by defined factors. Cell 131:861-872

53. Yu J, Vodyanik MA, Smuga-Otto K, Antosiewicz-Bourget J, Frane JL et al (2007) Induced pluripotent stem cell lines derived from human somatic cells. Science 318:1917-1920

54. Park IH, Zhao R, West JA, Yabuuchi A, Huo H et al (2008) Reprogramming of human somatic cells to pluripotency with defined factors. Nature 451:141-146

55. Thompson A, Zhang Y, Kamen D, Jackson CW, Cardiff RD et al (1996) Deregulated expression of c-myc in megakaryocytes of transgenic mice increases megakaryopoiesis and decreases polyploidization. J Biol Chem 271:22976-22982

56. Thompson A, Zhao Z, Ladd D, Zimmet J, Ravid K (1996) A new transgenic mouse model for the study of cell cycle control in megakaryocytes. Stem Cells 14(Suppl 1):181-187
57. Chanprasert S, Geddis AE, Barroga C, Fox NE, Kaushansky K (2006) Thrombopoietin (TPO) induces c-myc expression through a PI3K- and MAPK-dependent pathway that is not mediated by Akt, PKCzeta or mTOR in TPO-dependent cell lines and primary megakaryocytes. Cell Signal 18:1212-1218

58. Guo Y, Niu C, Breslin P, Tang M, Zhang S et al (2009) c-Mycmediated control of cell fate in megakaryocyte-erythrocyte progenitors. Blood 114:2097-2106

59. Vyas P, Ault K, Jackson CW, Orkin SH, Shivdasani RA (1999) Consequences of GATA-1 deficiency in megakaryocytes and platelets. Blood 93:2867-2875

60. Nishimura K, Segawa H, Goto T, Morishita M, Masago A et al (2007) Persistent and stable gene expression by a cytoplasmic RNA replicon based on a noncytopathic variant Sendai virus. J Biol Chem 282:27383-27391

61. Geddis AE (2011) Congenital amegakaryocytic thrombocytopenia. Pediatr Blood Cancer 57:199-203

62. Takayama NHS, Jono-Ohnishi R, Nakamura S, Hirose S, Endo H, Nishi M, Hamazaki Y, Kaneko S, Ishii E, Nakauchi H, Kunishima S, Eto K (2011) Modeling congenital amegakaryocytic thrombocytopenia using patient-specific induced pluripotent stem cells. Blood 118:703

63. Schiffer CA (2001) Diagnosis and management of refractoriness to platelet transfusion. Blood Rev 15:175-180

64. Ruggeri ZM (2002) Platelets in atherothrombosis. Nat Med $8: 1227-1234$

65. Nieswandt B, Watson SP (2003) Platelet-collagen interaction: is GPVI the central receptor? Blood 102:449-461

66. Canobbio I, Balduini C, Torti M (2004) Signalling through the platelet glycoprotein Ib-V-IX complex. Cell Signal 16:1329-1344

67. Rabie T, Strehl A, Ludwig A, Nieswandt B (2005) Evidence for a role of ADAM17 (TACE) in the regulation of platelet glycoprotein V. J Biol Chem 280:14462-14468

68. Bergmeier W, Burger PC, Piffath CL, Hoffmeister KM, Hartwig $\mathrm{JH}$ et al (2003) Metalloproteinase inhibitors improve the recovery and hemostatic function of in vitro-aged or -injured mouse platelets. Blood 102:4229-4235

69. Darrow AL, Fung-Leung WP, Ye RD, Santulli RJ, Cheung WM et al (1996) Biological consequences of thrombin receptor deficiency in mice. Thromb Haemost 76:860-866

70. Ishihara H, Zeng D, Connolly AJ, Tam C, Coughlin SR (1998) Antibodies to protease-activated receptor 3 inhibit activation of mouse platelets by thrombin. Blood 91:4152-4157

71. Terashita Z, Imura Y, Nishikawa K (1985) Inhibition by CV3988 of the binding of $[3 \mathrm{H}]$-platelet activating factor $(\mathrm{PAF})$ to the platelet. Biochem Pharmacol 34:1491-1495

72. Muller AM, Medvinsky A, Strouboulis J, Grosveld F, Dzierzak E (1994) Development of hematopoietic stem cell activity in the mouse embryo. Immunity 1:291-301

73. Medvinsky A, Dzierzak E (1996) Definitive hematopoiesis is autonomously initiated by the AGM region. Cell 86:897-906

74. Samokhvalov IM, Samokhvalova NI, Nishikawa S (2007) Cell tracing shows the contribution of the yolk sac to adult haematopoiesis. Nature 446:1056-1061

75. Schulze H, Shivdasani RA (2005) Mechanisms of thrombopoiesis. J Thromb Haemost 3:1717-1724

76. Deutsch VR, Tomer A (2006) Megakaryocyte development and platelet production. Br J Haematol 134:453-466

77. Gekas C, Rhodes KE, Gereige LM, Helgadottir H, Ferrari R et al (2009) Mef2C is a lineage-restricted target of Scl/Tall and regulates megakaryopoiesis and B-cell homeostasis. Blood 113:3461-3471

78. Park IH, Arora N, Huo H, Maherali N, Ahfeldt T et al (2008) Disease-specific induced pluripotent stem cells. Cell 134:877-886 